\title{
Gemstone Spectral Monochromatic CT Imaging: Detection and Classification of Focal Liver Lesions in Patients with Pancreatic Cancer
}

\author{
${ }^{1}$ Hu Shengping, ${ }^{1}$ Wang Mingliang, ${ }^{1}$ Wu Zhiyuan, ${ }^{1}$ Zhao Xuesong, ${ }^{2}$ Miao Fei \\ ${ }^{1}$ Shanghai Jiao Tong University, School of Medicine, Shanghai, China \\ ${ }^{2}$ Ruijin Hospital, Shanghai Jiao Tong University, School of Medicine, Shanghai, China
}

Correspondence: Miao Fei, Department of Radiology, Ruijin Hospital, Shanghai Jiao Tong University School of Medicine No. 197 Ruijin 2nd Road, Shanghai-200025, China, Phone: +86-21-64370045-665781, e-mail: mf11066@rjh.com.cn

\begin{abstract}
Objective: To analyze the diagnostic performance of gemstone spectral monochromatic CT imaging in the detection and classification of focal liver lesions in patients with pancreatic cancer.

Methods: We retrospectively studied 32 pancreatic cancer patients with 55 focal liver lesion images ( 19 cysts and 36 metastases) conducted by gemstone spectral CT. We obtained the focal liver lesions and the pancreatic iodine concentration from the iodine-based material decomposition images. This was further normalized to that of aorta. Receiver-operator characteristic curves to determine the diagnostic sensitivities and specificities of correctly diffentiating liver metastases from cysts were calculated. Imaging results were correlated with findings of intraoperative surgical $(n=13)$, imaging follow-up, ultrasound findings and percutaneous ethanol therapy $(n=19)$.

Results: Gemstone spectral CT played a significant role in differentiating focal liver metastases from liver cysts. The parameters we drew could provide extra quantitative data.

Conclusion: The gemstone spectral monochromatic CT scanner provides not only the processed polychromatic images, including arterial and portal phase images, but also extra quantitative data.

Abbreviations: CT: Computed tomography; GSI: Gemstone spectral imaging; MD: Material decomposition; HDCT: Helical dynamic CT, AP: Arterial phase; PP: Portal phase; ROC: Receiver operating characteristics; MDCT: Multidetector CT

Keywords: Gemstone spectral CT, GSI CT, Focal liver lesions, Pancreatic cancer.
\end{abstract}

\section{INTRODUCTION}

Pancreatic cancer is well-known for its high invasion and extremely poor prognosis. ${ }^{1,2}$ Surgical resection offers the best chance of treatment. However, liver metastases are frequently found at the time of diagnosis, thus losing the opportunity of surgical intervention. In addition, operation on patient with undiagnosed liver metastases may increase the unnecessary pain and economic burden and even bring worse prognosis to the patient. Thus, the detection of liver metastases is crucial for therapeutic planning and imaging which has to provide precise diagnosis to achieve optimal medical management for each patient. ${ }^{3}$ As a noninvasive method, conventional multislice computed tomography (CT) with dual phase imaging in arterial and portal venous phases has been widely used for diagnosing suspected pancreatic neoplasms, evaluating infiltration and lymph node metastases ${ }^{4}$ and differentiating benign from metastatic liver lesions. ${ }^{5}$ However, CT examination only represents the average Hounsfield value due to the partial volume effect and mixture of multiple photon energies may result in the reduction of the diagnostic value, especially for small focal lesions. ${ }^{6}$

Recently, a new CT scan mode based on a gemstone detector and related detection system, and reconstruction or gemstone spectral imaging (GSI) was introduced to provide CT spectral imaging. This scan mode is based on the simultaneous acquisition of two data sets, high energy and low energy, which allows precisely registered data sets to create accurate material decomposition (MD) images (e.g. the water and iodine-based images) and monochromatic spectral images with energy ranging from 40 to $140 \mathrm{keV}$ throughout the full $50 \mathrm{~cm}$ field of view. Therefore, the purpose of this study was to investigate the role of GSI in differentiation of small liver metastases from small benign lesions.

\section{MATERIALS AND METHODS}

\section{Patients}

The protocol for this study was approved by the institutional review board at our institution and informed consent for this retrospective study was acquired. From February to 
December in 2010, we reviewed 43 pancreatic cancer patients with focal liver lesions. GSI examination was performed for these patients. The patients without sufficient confirmation of the nature of the lesions $(n=11)$ were excluded. Therefore, the final study population was consisted of 32 pancreatic cancer patients (21 males; 11 females; mean age: 62.56 years; age range: $45-83$ years). Among these 32 cases, 18 patients had pancreatic head cancer, five patients had ampulla cancer and nine patients had distal bile duct cancer. The liver lesions with a diameter of less than $3 \mathrm{~cm}$ were included. The mean diameter of the liver lesion was $1.73 \mathrm{~cm}$ ranging from 0.5 to $2.9 \mathrm{~cm}$. The maximum number of the lesions in one patient was limited to five. Liver metastasis was confirmed by surgery in 11 cases, needle aspiration biopsy in two cases and followup $^{17}$ F-FDG PET/CT, ultrasound or CT/MRI scanning examination in 19 cases. Among the 11 surgery-confirmed cases, seven cases were confirmed by histological analysis and four cases were confirmed by palpation during the operation.

\section{Scanning Parameters}

Discovery CT750HD scanner was applied to all patients. First, unenhanced helical scans were acquired with a collimation of $5 \mathrm{~mm}$, a pitch of $1: 3$ and a reconstruction interval of $5 \mathrm{~mm}, 120 \mathrm{kVp}$ and $150 \mathrm{~mA}$. Then, all patients were injected with nonionic contrast medium (Optiray 320, Montreal, Canada) through antecubital venous at a rate of 3 to $4 \mathrm{ml} / \mathrm{sec}$ for a total of 90 to $120 \mathrm{ml}$ ( $1.5 \mathrm{ml} / \mathrm{kg})$. Finally, dual-phase scanning was performed with GSI mode: Spectral with fast tube voltage switching between 80 and $140 \mathrm{kVp}$ on adjacent views during a single rotation, 0.625 mm collimation, 0.6 second gantry rotation speed and 0.984:1 helical pitch. Images were reconstructed with a helical dynamic CT (HDCT) imaging reconstruction. From the single GSI, we can acquire the conventional polychromatic images, the iodine and water-based MD images, and a set of monochromatic images with energy ranging from 40 to $140 \mathrm{keV}$. Late arterial phase imaging was initiated within 10 seconds after enhancement of the descending aorta to $120 \mathrm{HU}$, as measured by a bolustracking technique (Smart Prep; GE Medical Systems). Portal venous phase images were obtained by using a 70second delay. The images were obtained in a craniocaudal direction from the top of the diaphragm to the lower pole of the kidney during a single breath-hold.

\section{GSI Analyses}

Both arterial phase (AP) and portal phase (PP) were acquired from each patient (Fig. 1). Serial attenuation measurements of the liver lesions, pancreatic lesions, aorta and liver parenchyma were obtained for three times. These measurements were acquired at the midpoint of each imaging with an elliptic or circular receiver operating characteristic (ROC). The size of the ROI was determined in each individual patient by aortic and lesion dimensions. A hepatic ROC was acquired from three different levels. The average value of these three measurements in each patient was obtained.

Iodine concentration ( $\mathrm{ICL}_{\mathrm{AP}}$, ICL $\mathrm{IP}_{\mathrm{PP}}$ ) of focal liver lesions and pancreatic lesions was obtained according to the iodinebased material decomposition images of liver and pancreatic cancer lesions, respectively (Fig. 2). The ICL value was further normalized (ICL/A) to arterial iodine concentration of aorta. Tumor-to-liver (T-to-L) contrast was calculated as ICL to liver parenchyma ratio, T-to-L was determined by the differences in attenuation measurements from ROI placed within the most enhanced portion of the neoplasm to the adjacent normal hepatic parenchyma (Fig. 2).
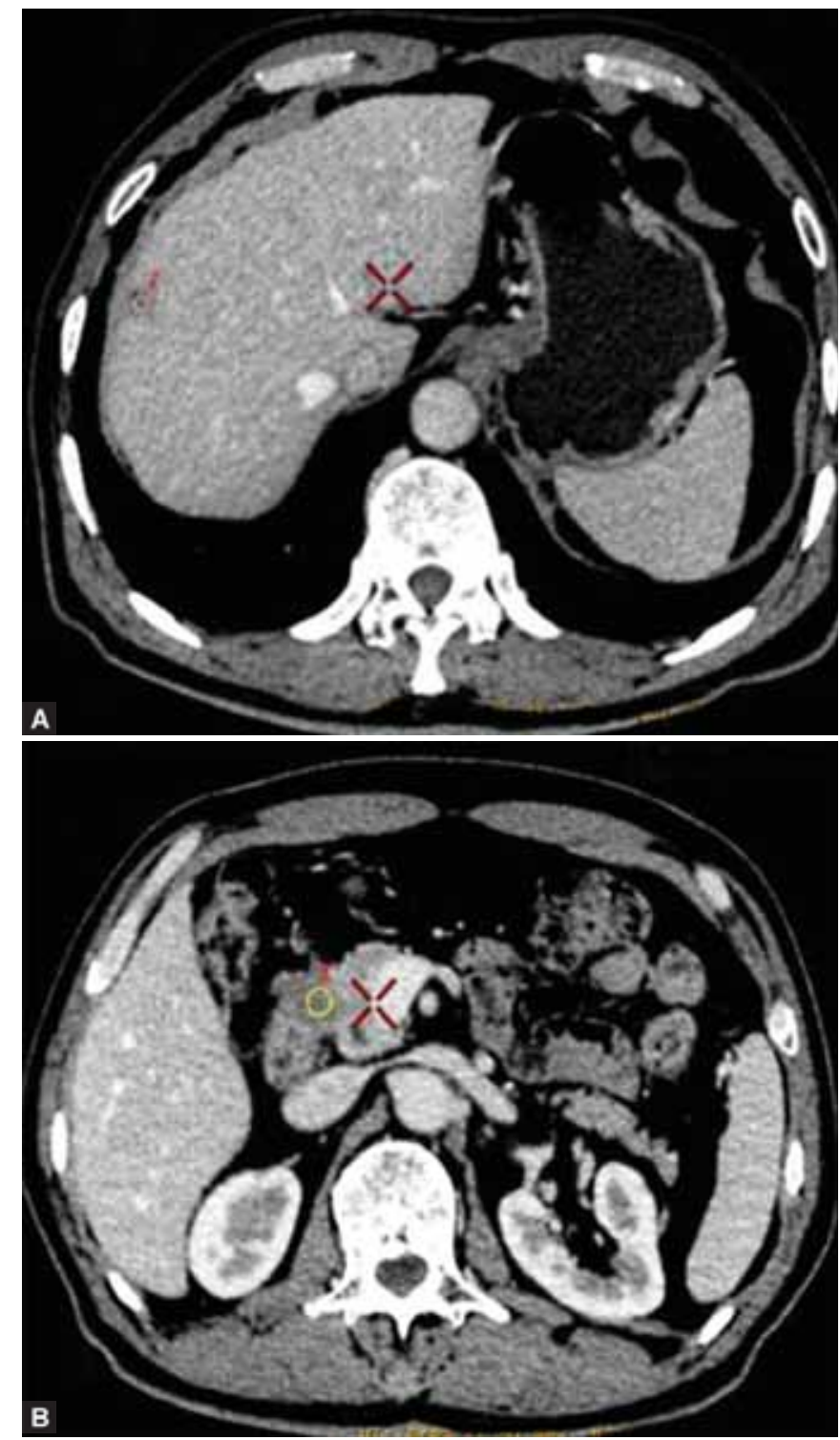

Figs $1 \mathrm{~A}$ and $\mathrm{B}$ : $\mathrm{RO}$ I for hepatic and pancreatic lesions in a patient within pancreatic cancer. A 70-year-old pancreatic cancer patient with hepatic metastases: (A) ROI for liver lesion, (B) ROI for pancreatic lesion 


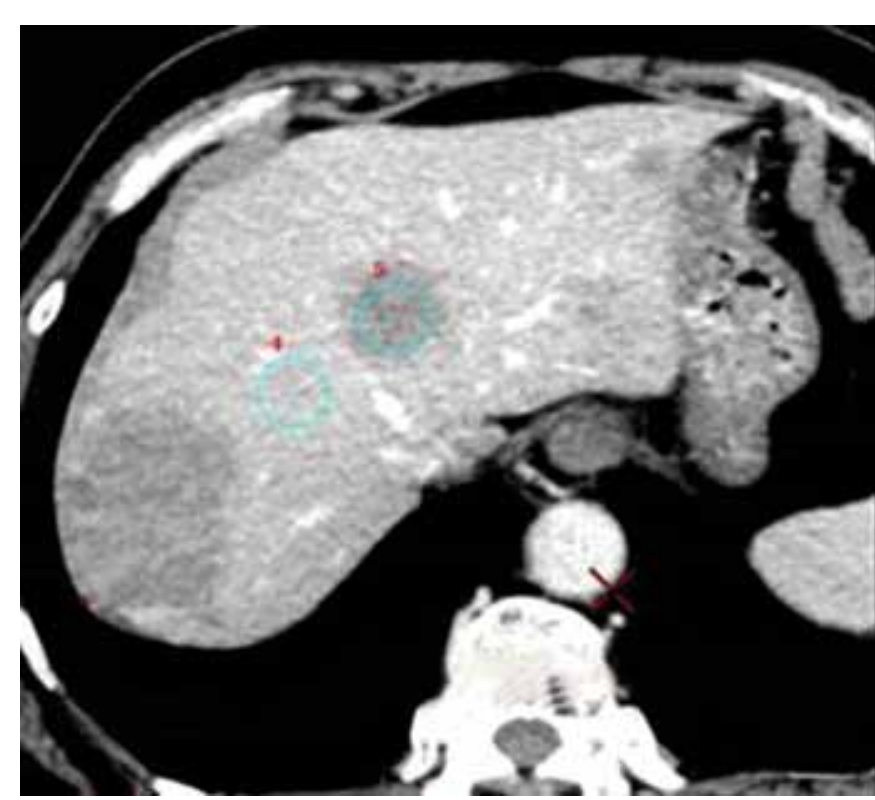

Fig. 2: Measurement of region of interest (ROI) of hepatic metastasis. A 76-year-old man with hepatic multi-metastases. Region-of-interest measurements were obtained from tumor (lable 5) and adjacent hepatic parenchyma (lable 4) at identical anatomic locations. GSI scanner showed hypointensed tumor to the background of liver parenchyma. Tumor-to-lesion was calculated

ROC curves $^{7}$ were generated to distinguish hepatic metastases from benign cysts. True-positive cases were defined as metastases that were correctly diagnosed. Falsepositive cases were defined as benign lesions that were incorrectly diagnosed as metastases. The diagnostic capability was determined by calculating the area under each reader-specific receiver operating characteristic curve (AUC) and AUC's p-value. The optimal sensitivity and specificity were defined as the maximum sensitivity with specificity.

\section{Statistical Analyses}

Statistical analysis was performed using SPASS13.0. $\mathrm{p}<0.05$ was considered statistically different. Student's t-test was performed to determine the difference between $\mathrm{ICL}_{\mathrm{A}_{\mathrm{AP}}, \mathrm{ICL}_{\mathrm{AP}} \text {, ICL }}$ PP ICP $_{\mathrm{AP}}, \mathrm{ICP}_{\mathrm{PP}}$ and tumor-to-liver in metastases and those in benign cyst.

\section{RESULTS}

All the parameters of hepatic metastases were higher than those of liver cysts. ICL/ $\mathrm{A}_{\mathrm{AP}}$ of liver metastases were 5fold higher than that of cysts. Both $\mathrm{ICL}_{\mathrm{AP}}$ and $\mathrm{ICL}_{\mathrm{PP}}$ of metastases were 2.5-fold higher than that of benign cyst. ICL $_{\mathrm{PP}}$ of metastases was significantly higher than that of cyst $(p=0.0456)$. Compared with other parameters, ICL $_{\mathrm{PP}}$ had less distinct difference between metastases and cysts. Significant difference was also observed between the maximum T-to-L contrast in AP for metastatic neoplasm and that for benign cyst ( $p=0.0059$ ) (Table 1). We also analyzed the correlation between $\mathrm{ICL}_{\mathrm{ap}}$ and $\mathrm{ICL}_{\mathrm{pp}}$ in the focal hepatic lesion and ICL in pancreatic ductal adenocarcinoma. The results showed that $\mathrm{ICL}_{\mathrm{ap}}$ in the focal hepatic lesion was significantly correlated with ICL in the pancreatic lesion ( $\mathrm{R}=0.8743$, $\mathrm{p}=0.0001$ ) which suggesting that hepatic metastasis was significantly correlated to the pancreatic lesion. $\mathrm{ICL}_{\mathrm{pp}}$ in the focal hepatic lesion was also significantly correlated with ICL in the pancreatic lesion ( $\mathrm{R}=0.843, \mathrm{p}=0.0001)$. In contrast, ICL in hepatic cysts was not significantly correlated with that in the pancreatic cancer (Table 2).

Results in Table 3 showed that T-to- $\mathrm{L}_{\mathrm{pp}}$ cannot be used for characterizing hepatic lesions $(p=0.514)$. ICL/A $\mathrm{A}_{\mathrm{AP}}$, $\mathrm{ICL}_{\mathrm{AP}}, \mathrm{ICL}_{\mathrm{PP}}$ and $\mathrm{T}$-to- $\mathrm{L}_{\mathrm{AP}}$ in malignant lesions were significantly different from those in the benign lesions which indicating that these parameters can be used for distinguishing benign lesions from malignant lesions.

\section{DISCUSSION}

The incidence of pancreatic cancer has been increasing recently, and the overall 5-year survival rate for patients with pancreatic adenocarcinoma was low. ${ }^{10}$ Recent advances in the chemotherapy have improved the survival of patients with pancreatic cancer. A 5-year overall survival rate after surgical resection of pancreatic adenocarcinoma has been reported to be $18 \% .{ }^{11}$ Despite the poor prognosis of patients with pancreatic cancer, surgical resection is still the only potentially curative treatment for the disease. However, surgical operation for the pancreatic cancer patients with hepatic metastases often brings unnecessary physical and economic losses. Therefore, early diagnosis and accurate assessment of the nature of focal liver lesions are important for diagnostic imaging.

Due to the high spatial definition, multidetector CT (MDCT) has been used as a key method to characterize abdominal lesions since its emergence. With increasing gantry speed and thinner scanner layer, the sensitivity of MDCT for detecting lesions is increasing in recent years. Bolus contrast material and Hounsfield value measurement provide a valid method to characterize lesions. Polychromatic enhanced CT images, including arterial and portal phase, were scanned to characterize hepatic lesions. Hepatic metastases were characterized on contrast-enhanced MDCT as globular or peripheral enhancement, a hypotensed lesion with circular hyperintense tissue around the lesion or having an obscure limit from the surrounding parenchyma. ${ }^{8}$ The typical feature of focal cyst on the fluid attenuation measurements was the appearance of round oval and no contrast-enhancement with well-defined borders. ${ }^{9}$ However, MDCT incorporated polychromatic X-ray 


\begin{tabular}{llllll} 
& \multicolumn{5}{c}{ Table 1: lodine concentration for different parameters } \\
& $I C L / A_{A P}$ & $I C L_{A P}$ & $I C L_{P P}$ & $T$-to- $L_{A P}$ & $T$-to- $L_{P P}$ \\
\hline Metastases & $0.20 \pm 0.31$ & $1.56 \pm 1.13$ & $2.16 \pm 1.21$ & $1.42 \pm 0.82$ & $0.66 \pm 0.19$ \\
Cysts & $0.04 \pm 0.03$ & $0.51 \pm 0.31$ & $0.84 \pm 0.63$ & $0.63 \pm 0.33$ & $0.28 \pm 0.21$ \\
p-value $(\mathrm{p}<0.05)$ & 0.004 & 0.001 & 0.0456 & 0.0059 & 0.6536 \\
\hline
\end{tabular}

Iodine concentration and p-value for the parameter of ICL/A $\mathrm{AP}_{\mathrm{AP}}$ ICL $\mathrm{L}_{\mathrm{PP}}, \mathrm{ICL}_{\mathrm{AP}}, \mathrm{T}$-to- $\mathrm{L}_{\mathrm{AP}}$ and T-to-L $\mathrm{L}_{\mathrm{PP}}, \mathrm{p}<0.05$

Table 2: Correlation analysis between hepatic focal lesions and pancreatic lesions in arterial and portal phase

\begin{tabular}{llll} 
& & Correlation coefficient & p-value (0.01) \\
\hline Metastases (pancreatic cancer) & AP & 0.8754 & 0.0001 \\
& PP & 0.843 & 0.0001 \\
Cysts (pancreatic cancer) & AP & 0.35428 & 0.3152 \\
& PP & 0.50242 & 0.1389 \\
\hline
\end{tabular}

\begin{tabular}{lllll}
\multicolumn{5}{c}{ Table 3: ROC curve analyzing, including sensitivity, specificity, area under curve } \\
& Sensitivity & Specificity & AUC (95\%) & $p$-value \\
\hline ICL $_{\mathrm{AP}}$ & 80 & 94.7 & $0.898(0.815-0.982)$ & 0.000 \\
ICL/A $_{\mathrm{AP}}$ & 82.1 & 95.2 & $0.898(0.815-0.980)$ & 0.000 \\
ICL $_{\mathrm{PP}}$ & 80 & 68.4 & $0.55(0.389-0.720)$ & 0.514 \\
T-to-L $_{\text {AP }}$ & 85.7 & 83.3 & $0.89(0.799-0.993)$ & 0.000 \\
T-to-L & 84.2 & $0.902(0.805-0.998)$ & 0.000 \\
\hline
\end{tabular}

collection technologies and its CT value was derived from the average photon energy (Fig. 3). The averaging effect from partial volume and mixture of multiple photon energies of tube voltage obscure the CT numbers, ${ }^{6}$ which affect the characterization of focal liver lesions, especially those with a small diameter (e.g. $<1.0 \mathrm{~cm}$ ). ${ }^{12,13}$ We have experienced the low sensitivity of MDCT in previous reports, especially when compared with MR. ${ }^{14}$ However, in clinical practice, patients with pancreatic cancer in most cases are undergoing MSCT as opposed to abdomen MR protocol due to both financial and logistic reasons. A new CT scanner has emerged recently. In the past 3 years, spectral imaging with gemstone detector material (GSI mode) emerges as the latest technology for CT spectral imaging. GSI is based on fast $\mathrm{kV}$ switching-dynamic switching between two different $\mathrm{X}$-rays (80 and $140 \mathrm{kVp}$ ) during a single rotation. Monochromatic imaging not only improves image quality ${ }^{15,16}$ and reduces image noise but also provides discrimination of different tissue types based on material density and monochromatic image data. GSI data can be segregated among a material density pair, e.g. water and iodine. They can switch between material pairs. In radiological imaging, water and iodine-based material is commonly used because it reflects the material composition and enhancement during CT imaging. Measuring the mg/ cc of iodine/water from material decomposition offers true CT number in assessment the material composition of liver

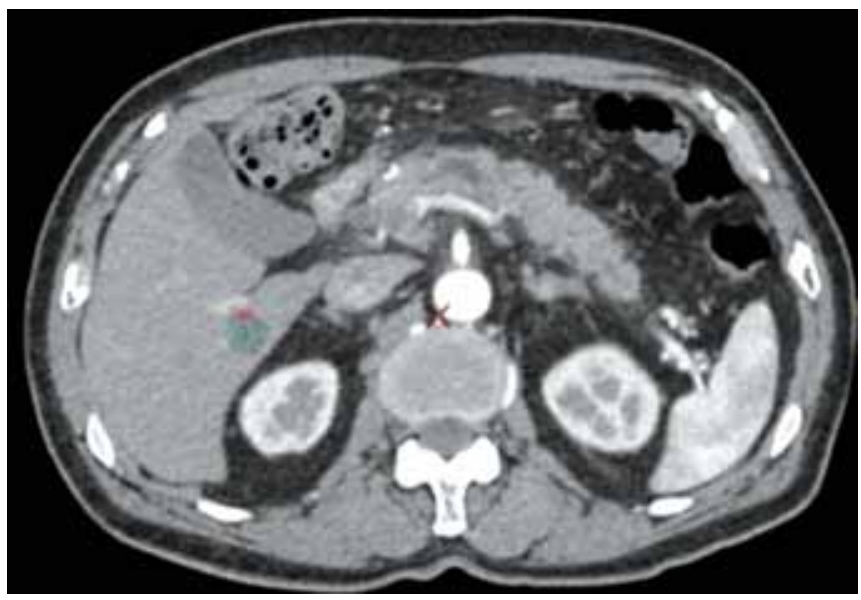

Fig. 3: Assessment of hepatic cyst. A 72-year-old man with hepatic cyst, small lesion difficult to characterize by MDCT

lesions, which provide a means to differentiate liver metastases from benign lesions. In MDCT, liver metastases are characterized by enhancement pattern and degree. Late arterial and portal phases are the optimal phases for detecting metastatic neoplasms. ${ }^{17,18}$ The spectral imaging can provide qualitative images as the common MDCT imaging. More importantly, iodine and water scatterplot in GSI-CT provide a visualized way to assess the material composition and distribution in the areas of interests (Fig. 4). Powerful postprocessing and reconstructing function of GSI spectral CT provide an extremely useful assistance to make precise radiological diagnosis. 

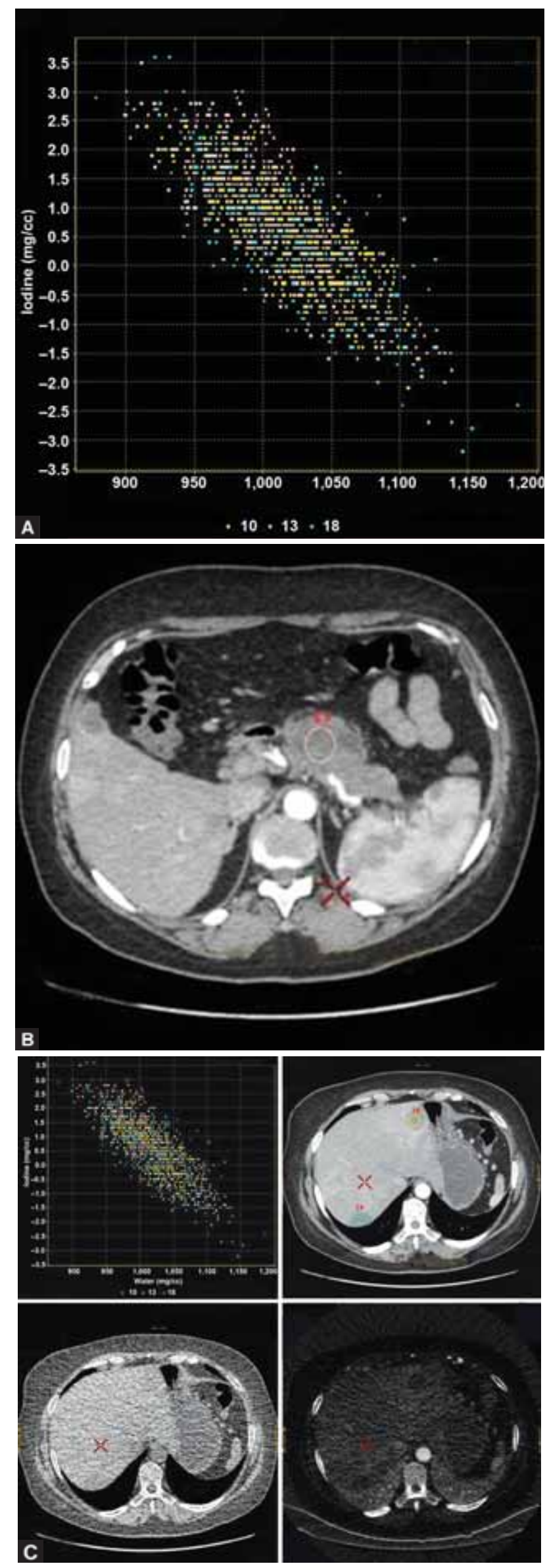

Figs 4A to C: GSI iodine and water scatterplot: (A) GSI iodine and water scatterplot, (B) ROI in pancreatic cancer, (C) ROI in liver lesions
Compared with conventional CT imaging in which the diagnosis is affected by the experience of the readers, the spectral imaging provides more objective and quantitative parameters that are less affected by the experience of the readers. To our knowledge, MDCT estimates enhancement based on the arterial and portal phases. We normalized the parameters ICL/A and compared these parameters with statistical analysis. In our study, three parameters ICL $_{\mathrm{AP}}$, ICL $_{\mathrm{PP}}$ and T-to-L $\mathrm{AP}_{\mathrm{AP}}$ (after normalization) had significant difference between liver lesions and benign cysts. Therefore, these parameters can be used alone for differentiation diagnosis. We also discovered the close correlation of material composition between hepatic metastases and pancreatic cancer. ICL in hepatic metastasis was significantly correlated to that in the pancreatic lesions. In contrast, ICL of hepatic cysts was not significantly correlated to that in the pancreatic lesions. This correlation analysis indicated that the hepatic metastases lesions had similar material composition to pancreatic cancers. The gemstone spectral monochromatic CT imaging can assist the radiologists to characterize focal liver lesions from both the qualitative and quantitative angles.

In addition to these advantages, our study still has some limitations. First, histopathologic confirmation and/or intraoperative surgery were only available in 18 of the 55 lesions (13 of 32 patients). Obtaining histopathological diagnosis in all patients would be desirable, but this is difficult due to both practical and particularly ethical reasons in many cases. Nevertheless, we obtained PET-CT, ultrasound and other imaging follow-up examinations to verify these results. Second, we excluded the benign liver lesions: Hemangioma, focal fatty infiltration and ignored the original liver tumors from our sample which might affect the results.

\section{CONCLUSION}

Gemstone spectral imaging not only provides material decomposition and monochromatic spectral imaging but also gives us several quantitative parameters in characterizing focal liver lesions. GSI scanner was superior in the detection and classification of focal liver lesions in patients with pancreatic cancer.

\section{ACKNOWLEDGMENT}

The authors thank Shanghai Science and Technology Committee for its valuable support.

\section{REFERENCES}

1. Jemal A, Siegel R, Ward E, et al. Cancer statistics 2008. CA Cancer J Clin 2008;58:71-96.

2. Wang L, Yang GH, Lu XH, Huang ZJ, Li H. Pancreatic cancer mortality in China (1991-2000). World J Gastroenterol 2003;9:1819-23. 
3. Patrick M, Sandra P, Gress Thomas M. Evidence-based diagnosis and staging of pancreatic cancer. Best Practice and Research Clinical Gastroenterology 2006;20(2):227-51.

4. Smith SL, Rajan PS. Imaging of pancreatic adenocarcinoma with emphasis on multidetector CT. Clin Radiol 2004;59:26-38.

5. Multidetector CT of pancreas. Effects of contrast material flow rate and individualized scan delay on enhancement of pancreas and tumor contrast. Radiology 2006;241:441-48.

6. Soo G, Lau K, Yik T, Kutschera P. Optimal reconstructed section thickness for the detection of liver lesions with multidetector CT. Clin Radiol 2010;65:193-97.

7. Zou Kelly H, O’Malley, A James, Mauri Laura. Predictive models receiver-operating characteristic analysis for evaluating diagnostic tests and predictive models. Circulation 2007;115;654-57.

8. Altekruse SF, Kosary CL, Krapcho M, et al (eds). SEER Cancer Statistics Review 1975-2007, National Cancer Institute. Bethesda, MD, based on November 2009 SEER data submission, posted to the SEER web site 2010.

9. Cameron JL, Riall TS, Coleman J, Belcher KA. One thousand consecutive pancreaticoduodenectomies. Ann Surg 2006; 244:10-15.

10. Leslie DF, Johnson CD, Johnson CM. Distinction between cavernous hemangiomas of the liver and hepatic metastases on CT: Value of contrast enhancement patterns. AJR Am J Roentgenol 1995;164:625-29.

11. Horton KM, Bluemke DA, Hruban RH, Soyer P, Fishman EK. $\mathrm{CT}$ and MR imaging of benign hepatic and biliary tumors.
Radiographics: A Review Publication of the Radiological Society of North America, Inc 1999;19(2):431-51.

12. Haider MA, Amitai MM, Rappaport DC, et al. Multi-detector row helical CT in preoperative assessment of small $(<\mathrm{or}=1.5$ $\mathrm{cm}$ ) liver metastases: Is thinner collimation better? Radiology 2002;225:137-42.

13. van LMS, Noordzij J, Feldberg MA, Hennipman AH, Doornewaard H. Focal liver lesions: Characterization with triphasic spiral CT. Radiology 1996;201:327-36.

14. Hammerstingl R, Huppertz A, Breuer J, et al. Diagnostic efficacy of gadoxetic acid (Primovist)-enhanced MRI and spiral CT for a therapeutic strategy: Comparison with intraoperative and histopathologic findings in focal liver lesions. Eur Radiol 2008;18:457-67.

15. Matsumoto K, Jinzaki M, Tanami Y, Ueno A, Yamada M, Kuribayashi S. Virtual monochromatic spectral imaging with fast kilovoltage switching: Improved image quality as compared with that obtained with conventional $120 \mathrm{kVp}$ CT. Radiology; 259:257-62.

16. Lin XZ, Miao F, Li JY, Dong HP, Shen Y, Chen KM. Highdefinition CT gemstone spectral imaging of the brain: Initial results of selecting optimal monochromatic image for beamhardening artifacts and image noise reduction. Comput Assist Tomogr 201;35:294-97.

17. Foley WD, Mallisee TA, Hohenwalter MD, et al. Multiphase hepatic CT with a multirow detector CT scanner [J]. AJR 2000; 175:679-85.

18. Laghi A. Multidetector CT (64 slices) of the liver. Examination techniques. Eur Radiol 2007,17:675-83. 